\title{
Robust Zonotopic Set-Membership Approach for Model-Based Prognosis: Application on Linear Parameter-Varying Systems
}

\author{
Ahmad Al-Mohamad ${ }^{1,2}$, Vicenç Puig ${ }^{1}$ and Ghaleb Hoblos ${ }^{2}$
}

\begin{abstract}
A robust set-membership Prognostics and Health Management (PHM) methodology is presented in this paper. The key advantages of the set-membership approach for states and parameters estimation are enhanced by employing zonotopes that are less conservative and computationally complex than other sets. The optimal tuning of the proposed observer is formulated using the Linear Matrix Inequality (LMI) approach. Moreover, the Joint Estimation of States and Parameters (JESP) leads to a non-linear representation of a monitored system that is transformed into a Linear Parameter-Varying (LPV) system by means of the non-linear embedding approach. The considered case study is based on a slowly degraded DC-DC converter. The aim of the proposed PHM approach is to forecast the Remaining Useful Life (RUL) on a system level. Additionally, the proposed RUL forecasting approach is independent of previous knowledge of the degradation behaviors being only dependent on the estimated zonotopic parameters. Finally, the obtained results demonstrate the efficiency of the proposed approach.
\end{abstract}

\section{INTRODUCTION}

Fault diagnosis and prognosis are powerful approaches for the health management assessment of many engineering applications. They require critical and reliable state observation whether for condition-based or predictive maintenance, and for RUL forecasting [1]. In this context, the contributions of this paper such as modeling and identification, robust JESP, and RUL forecasting are all PHM-related. Specifically, we proposed the general scheme of the aforementioned threefold in [7], that has been adapted to the set-membership framework in this paper. Thus, the proposed PHM approach aims to achieve a reliable RUL forecasting which is mainly based on parameter estimation. For this reason, we intend to employ the robustness of the set-membership approach for JESP for the sake of RUL forecasting, considering unknown-but-bounded noises and uncertainties in an LPV framework.

In broad, PHM applications target dynamical systems with varying parameters. Therefore, parameters augmentation

This work is partially funded by the Catalan Agency for Management of University and Research Grants (AGAUR) and the European Social Fund (ESF) of the Government of Catalonia through the grant FI-SDUR-AGAUR 2020 (ref. 2020-FISDU-307). It is also partially funded by European Union and Normandy Region. Europe is involved in Normandy through the European Funds for Regional Development.

${ }^{1}$ Ahmad Al-Mohamad and Vicenç Puig are with Automatic Control Department, Universitat Politècnica de Catalunya (UPC), Campus de Terrassa, Rambla Sant Nebridi, 10, 08222, Spain ahmad.al.mohamadeupc.edu, vicenc.puig@upc.edu

${ }^{2}$ Ahmad Al-Mohamad and Ghaleb Hoblos are with Normandy University, UNIROUEN, ESIGELEC, IRSEEM, 76000, Rouen, France ahmad.al-mohamadeuniv-rouen.fr, ghaleb.hoblos@esigelec.fr is necessary for the JESP as investigated in [7]. But, this nonlinear representation may not guarantee the observability of the system when transforming the nonlinear representation into an LPV model. Since the time-varying elements depend on measurable parameters, the LPV is considered as a particular class of Linear Time Variant (LTV) systems. Technically, there exists more than a single methodology to identify and classify the LPV systems such as nonlinear embedding and linearization approximation [8]. In this case, we approximated the nonlinear model with multiple varying parameters using Jacobian linearization, and the LPV is represented in a polytopic form with bounded parameters.

The efficiency of the proposed PHM approach relies on the parameters estimation which has been carried out using the set-membership approach. Additionally, in spite of the existence of various geometrical shapes such as polytopes (including zonotopes, parallelotopes and boxes) and ellipsoids that could be integrated into the set-membership scheme, we adopted the zonotopes on account of the efficient arithmetic operations they provide, in addition to the reduced conservatism compared to other set representations [5], [6]. Therefore, the deterministic Zonotopic Set-Membership (ZSM) approach assumes unknown-but-bounded noises and disturbances [2]. It is worth mentioning that the ZSM and the interval observers approaches have been proved to be similar from a structural aspect under some conditions [3].

The ZSM observer design is an essential element that in this paper has been addressed using an optimization-based scheme to cope with the reliable JESP. In particular, a classical online approach based on the minimization of the $F$-radius with an optimality criterion ([10], [6]) has been compared to the proposed offline LMI-based $F_{\mathrm{w}}$-radius optimization.

Finally, a RUL forecasting approach has been developed based on a polynomial estimation of the End of Life (EoL) of the varying parameters, without previous knowledge of the degradation behaviors. The complete methodology has been assessed using a DC-DC buck-boost converter used in electric vehicles [7]. Thus, a system-level degradation has been modelled and investigated relying on two crucial power electronic components that are subject to harsh degradation. The cascading impact has been estimated using the ZSM for states and parameters. The system-level prognostics with multiple varying parameters that are characterized by different degradation behaviors are being developed, whilst the forecasted RUL of First Critical Component to Fail (FCCF) is considered as the RUL of the whole system on 
the macro level.

This paper is structured as follows. Some background material about zonotopes are provided in Section II. Thus, the key features of the proposed PHM approach are highlighted in Section III. The ZSM approach is demonstrated in Section IV. Next, the RUL forecasting approach is detailed in Section V. Section VI is dedicated to the application case study presenting simulation results. Finally, the conclusions are drawn in Section VII.

\section{BACKGROUND MATERIAL}

As discussed in the introduction, in this paper we will use zonotopes for the JESP in a set-membership framework. In the following, some background material regarding zonotopes is introduced.

Definition 2.1 (Zonotopes): A zonotope $\mathcal{Z} \subset \mathbb{R}^{n}$ is a convex symmetric polytope defined by a linear image of a hypercube $[-1,+1]^{m} \subset \mathbb{R}^{m}$, with a center $c \subset \mathbb{R}^{n}$ and a generator matrix $H \subset \mathbb{R}^{n \times m}$. It can be expressed as:

$$
\mathcal{Z}=\langle c, H\rangle=\left\{c+H h,\|h\|_{\infty} \leq 1\right\}
$$

Property 2.1 (Minkowski Sum): A p-order zonotope $\mathcal{Z}$ can be expressed as $\mathcal{Z}=c \oplus H \mathbf{B}^{p}$, where $\oplus$ denotes the Minkowski sum. Then, the Minkowski sum of two zonotopes $Z_{1}=\left\langle c_{1}, H_{1}\right\rangle$ and $Z_{2}=\left\langle c_{2}, H_{2}\right\rangle$ is:

$$
\mathcal{Z}_{1} \oplus \mathcal{Z}_{2}=\left(c_{1}+c_{2}\right) \oplus\left[H_{1}, H_{2}\right] \mathbf{B}^{p_{1}+p_{2}} .
$$

Property 2.2 (Reduction operator): The weighted reduction operator $\downarrow_{\mathrm{q}, \mathrm{w}}$ is used to reduce the number of vertices of the generator matrix $H$, where $q$ specifies the maximum number of columns in the reduced generator matrix as $\downarrow_{\mathrm{q}, \mathrm{w}} H$ [6].

Definition $2.2\left(F_{w}-\right.$ radius $): F_{\mathrm{w}}$-radius is an effective size criterion for zonotopes $\mathcal{Z}=\langle c, H\rangle \subset \mathbb{R}^{n}$. It is calculated by a weighted Frobenius norm of $H$ as [6]:

$$
\|\langle c, H\rangle\|_{\mathrm{F}, \mathrm{w}}=\|H\|_{\mathrm{F}, \mathrm{w}} .
$$

Definition 2.3 ( $F$-radius): The $F$-radius criterion is similar to the $F_{\mathrm{w}}$-radius with $\mathrm{w}=I_{n}$. Thus, the Frobenius norm of $\mathcal{Z}=\langle c, H\rangle \subset \mathbb{R}^{n}$ is computed as [6]:

$$
\|\langle c, H\rangle\|_{F}=\|H\|_{F},
$$

\section{PROPOSED PROGNOSIS AND HEALTH MANAGEMENT APPROACH}

Nowadays, it has become crucial to observe and assess the state of critical systems. The health monitoring requires various clusters of information such as measurements data, estimated data, human interface and more, while the advanced techniques focus on the application of the predictive maintenance. In particular, the proposed approach deals with degraded systems such as power electronic systems due to thermal and/or electrical overstresses.

To date, the ongoing research targeting reliable RUL forecasting is still challenging. The reasons behind these difficulties refer to the wide variety of applications, the modeling complexity of the systems, the working conditions, the accessible measurements, the types of the components, and more reasonable constraints. Despite that, some of these disciplines could be classified into clusters to manage the structuring of the most suitable PHM approach for a specific application. Interestingly, the model-based systems could follow the same strategy that leads to a satisfactory RUL forecasting. Thus, the key points of the proposed PHM approach are illustrated in Figure 1, and provided with explanations for RUL forecasting of model-based dynamical systems with slow degraded parameters. Finally, it is worth mentioning that one of the significant features of this proposed approach is its ability to forecast the RUL without previous knowledge of the degradation behaviors [7].

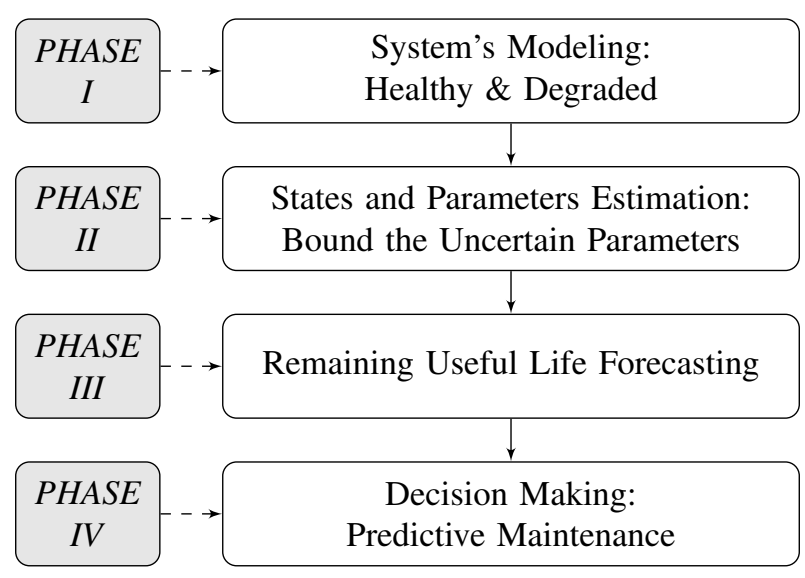

Fig. 1. Proposed PHM Approach

- Phase I: The dynamical model is considered with nonlinear degraded components in an augmented representation. Thus, for generalization purposes of such applications, the LPV representation has been adopted. Moreover, DC-DC converters are usually modelled as switched systems, yet the average representation is applied due to its reduced computational effort. It is worthwhile noting that the degradation behaviors are only utilized as references to simulate the degraded scenario, and to compare the zonotopic JESP with the empirical degradation models. Hence, the ZSM for JESP can now be applied the LPV model with multiple varying parameters.

- Phase II: Several methods could be employed for the states and parameters estimation in such dynamical systems. However, some constraints might impose specific techniques over others due to their advancement in observers tuning. In broad, the observability is crucial for the estimation, yet the problem turns to be harder while augmenting the model with more varying parameters and keeping less measurements data. More constraints are considered such as unknown-butbounded uncertainties with variable inputs, and the aim is to guarantee its observability. The failure condition is then attained when the degraded parameters exceed their physical thresholds that have been predefined based on accelerated aging experiments. 
In summary, the degradation estimation plays the most important role in the proposed PHM approach. Hence, as much as the complexity of the model increases with the aforementioned constraints, as much as the efficiency of the RUL forecasting decreases. Consequently, bounding the uncertain states and parameters has been utilized to guarantee a robust base for the following phase of RUL forecasting.

- Phase III: The online RUL forecasting is one of the main contributions to this study to ensure fast and reliable prediction. This approach employs sets of estimated parameters in zonotopic forms in order to generate intervals of polynomial EoL, and compute the RUL of each varying parameter.

- Phase IV: The last phase of this PHM approach depends on the type of application. Human-machine interface could be required for maintenance while in different situations, maintenance rescheduling would be a best fit. In broad, cost optimization by avoiding unnecessary maintenance is an essential financial target. Technically, degraded components damage the healthy ones, and this situation of cascading degradation can be avoided with further investigations.

\section{ZONOTOPIC SET-MEMBERSHIP FOR STATES AND PARAMETERS ESTIMATION}

\section{A. Problem Set-up}

Consider the following discrete-time state-space representation of an uncertain LPV model:

$$
\begin{aligned}
x_{k+1} & =A\left(\rho_{k}\right) x_{k}+B\left(\rho_{k}\right) u_{k}+E_{\omega} \omega_{k}, \\
y_{k} & =C\left(\rho_{k}\right) x_{k}+D\left(\rho_{k}\right) u_{k}+F_{v} v_{k} .
\end{aligned}
$$

where $k \in \mathbb{N}$ denotes the discrete time instant. $A_{k}=$ $A\left(\rho_{k}\right)=A_{0}+\Delta A\left(\rho_{k}\right)$ and similarly for the rest of the state-space matrices. $A_{k} \in \mathbb{R}^{n_{x} \times n_{x}}, B_{k} \in \mathbb{R}^{n_{x} \times n_{u}}, C_{k} \in$ $\mathbb{R}^{n_{y} \times n_{x}}$ and $D_{k} \in \mathbb{R}^{n_{y} \times n_{u}}$ denote the state matrix, input matrix, output matrix and feed-through matrix respectively. Additionally, $x_{k} \in \mathbb{R}^{n_{x}}, y_{k} \in \mathbb{R}^{n_{y}}$ and $u_{k} \in \mathbb{R}^{n_{u}}$ are the states, outputs and inputs of the system respectively. Moreover, $\omega_{k} \in \mathbb{R}^{n_{x}}$ and $v_{k} \in \mathbb{R}^{n_{y}}$ are the process disturbances and measurement noises respectively, with $E_{w} \in \mathbb{R}^{n_{x} \times n_{x}}$ and $F_{v} \in \mathbb{R}^{n_{y} \times n_{y}}$ identify their direction matrices.

Assumption 4.1: The disturbances and noises are assumed to be unknown-but-bounded zonotopes as:

$$
\mathcal{W}=\omega_{c} \oplus E_{\omega} \mathbf{B}^{n_{x}}, \quad \text { and } \quad \mathcal{V}=v_{c} \oplus E_{v} \mathbf{B}^{n_{y}},
$$

where, $\omega_{k}$ and $v_{k}$ are bounded by a unitary hypercube centered at 0 as:

$$
\omega_{k} \in\left\langle 0, I_{n_{\omega}}\right\rangle, \quad \text { and } \quad v_{k} \in\left\langle 0, I_{n_{v}}\right\rangle, \quad \forall k \geq 0,
$$

\section{B. ZSM Approach}

Three steps are required for the JESP in the ZSM framework. Firstly, the centers and the radii of the zonotopes are predicted in uncertain ranges at the current time instant. Secondly, a strip is computed based on the previous time instant measurement unlike the stochastic approaches such as Kalman filters [9]. Thirdly, the strip intersects with the predicted uncertain states and parameters in order to estimate the certain zonotopes. It should be noted that multi-output systems require to compute a strip for each output, and to intersect with the predicted states and parameters sequentially for a guaranteed zonotopic enclosure and estimation [10]. The estimated zonotopic states and parameters after each intersection are represented as follows:

$$
\hat{\mathcal{X}}_{k+1}=\hat{c}_{k+1} \oplus \hat{H}_{k+1} \mathbf{B}^{\mathbf{m}}
$$

with,

$$
\begin{aligned}
\hat{c}_{k+1} & =\hat{c}_{k}+\Lambda\left(Y_{k}-\hat{y}\right), \\
\hat{H}_{k+1} & =\left[\begin{array}{lll}
\left(I-\Lambda C_{k}\right) \bar{H}_{k}, & \left(I-\Lambda C_{k}\right) E_{\omega}, & -\Lambda F_{v}
\end{array}\right],
\end{aligned}
$$

where $\hat{c}$ is the estimated center of the zonotope $\hat{\mathcal{X}}$, and $\hat{H}$ is its estimated generator matrix. $\bar{H}_{k}$ denotes the reduced matrix which is computed by applying the reduction operator as shown in Property 2.2, and $\mathbf{B}^{\mathbf{m}}$ is a unitary box. $I$ is an identity matrix with proper dimensions and $\Lambda$ is the tuning matrix. Moreover, $y_{k}$ and $\hat{y}_{k}$ denote the measured and the estimated outputs respectively.

$$
\hat{y}_{k}=\left(C_{k} \hat{c}_{k}+D_{k} u_{k}\right) .
$$

The estimated zonotope in Eq. (8) is realized by intersecting the predicted states and parameters with the measurement strip.

The uncertain prediction $\hat{P}$ is computed once at each time instant as:

$$
\hat{P}_{k+1}=\hat{x}_{k+1} \oplus \hat{H}_{k+1} \mathbf{B}^{\mathbf{m}},
$$

where,

$$
\begin{aligned}
\hat{x}_{k+1} & =A_{k} \hat{x}_{k}+B_{k} u_{k}+E_{\omega} \omega_{k}, \\
\hat{H}_{k+1} & =A_{k} \hat{H}_{k} .
\end{aligned}
$$

Furthermore, the strip $\mathfrak{X}_{y}$ is computed for each output alone based on the previous measurement as:

$$
\mathfrak{X}_{y}=\left\{x \in \mathbb{R}^{n_{x}}:\left|C_{k} x_{k}+D_{k} u_{k}-Y_{k}\right|<v_{k}\right\} .
$$

Finally, the intersection is realized, and the estimated certain zonotopic states and parameters are represented as follows:

$$
\left\langle\hat{c}_{k+1}, \hat{H}_{k+1}\right\rangle=\hat{P}_{\mathrm{k}+1} \cap \mathfrak{X}_{y}=\hat{c}_{k+1}(\Lambda) \oplus \hat{H}_{k+1}(\Lambda) \mathbf{B}^{\mathbf{m}} .
$$

\section{Computation of Tuning Matrix $\Lambda$}

The ZSM observer is designed by tuning the matrix $\Lambda$ as shown in Equations (9). The generator matrix of the zonotope is affected by the uncertainties which could negatively affect the size of the zonotopes and lead to uncertain estimation. Thus, the role that $\Lambda$ plays in guaranteeing tight enclosures of bounds is significantly crucial. Moreover, $\Lambda$ could be computed online using a classical method which is based on the minimization of the $F$-radius with an optimality criterion. On the other hand, an offline LMI-based optimization problem can be solved to minimize the $F_{\mathrm{w}}$-radius of the estimated zonotopes. The optimal computation of both approaches are explained below: 
1) LMI-based optimization of $\Lambda$ (offline):

Proposition 4.2: An LMI-based optimization problem has been proposed to compute the optimal tuning matrix denoted by $\Lambda_{\mathrm{LMI}}^{*}$. The objective is to minimize the $F$-radius of the zonotope $\mathcal{X}$ less than a positive scalar $\gamma$. The LMIs has been derived based on the stability Lyapunov theory. Therefore, the optimal tuning matrix $\Lambda_{\mathrm{LMI}}^{*}$ is obtained for the feasible solutions $\Gamma$ and $W$ as:

$$
\Lambda_{\mathrm{LMI}}^{*}=\Gamma^{-1} W .
$$

Hence, the LMIs have been derived from the Lyapunov function with respect to the $F_{\mathrm{w}}$-radius. Next, by applying Schur complement, the resulting LMI problem is formulated as:

$$
\begin{aligned}
& \underset{W, \Gamma}{\operatorname{minimize}} \gamma \\
& \text { subject to } \\
& {\left[\begin{array}{cc}
\gamma I & I \\
I & \Gamma
\end{array}\right] \succeq 0,} \\
& {\left[\begin{array}{cccc}
-\Gamma & \Gamma A-W C & \Gamma Q^{T} & W \\
\star & -\Gamma & 0 & 0 \\
\star & \star & -I & 0 \\
\star & \star & \star & -R^{-1}
\end{array}\right] \preceq 0}
\end{aligned}
$$

where $Q=\sqrt{E_{\omega}}$ and $R=F_{v}$ are the weighting parameters for tuning, and $\star$ denotes symmetrical elements. It should be noted that the derivation of (16) is not detailed due to size limitations.

The proposed LMI-based optimization problem can be solved offline to reduce the time and the computational effort that could lead to estimation delays if it were solved online. Moreover, the varying parameters in the LPV model are the degradation precursors of physical parameters. Thus, the variation of each parameter is bounded between its rated value $\rho_{0}$ and its physical threshold $(\mathrm{TH}) \rho_{\mathrm{TH}}$ that describes the EoL of the system, as shown below:

$$
\Delta \rho=\rho_{\max }-\rho_{\min }=\rho_{\mathrm{TH}}-\rho_{0},
$$

It is worth noting that the $\mathrm{TH}$ of the parameters are defined by excessive accelerated aging tests which characterize the probability of failures of the components. The online measurements of the degradation scenarios are provided by the online repositories in [13], [14].

The polytopic representation of (5) is obtained using the bounding box approach and considering the range of variation of the varying parameters as:

$$
\begin{gathered}
x(k+1)=\sum_{i=1}^{N} \mu_{i}\left(\rho_{k}\right)\left(A_{i} x(k)+B_{i} u(k)\right), \\
y(k)=\sum_{i=1}^{N} \mu_{i}\left(\rho_{k}\right)\left(C_{i} x(k)+D_{i} u(k)\right) .
\end{gathered}
$$

Next, a varying value for the observer gain (15) can obtained as follows:

$$
\Lambda_{\mathrm{LMI}^{*}}^{*}\left(\rho_{k}\right)=\sum_{i=1}^{N} \mu_{i}\left(\rho_{k}\right) \Lambda_{\mathrm{LMI}_{i}}^{*}
$$

where $\Lambda_{\mathrm{LMI}_{i}}^{*}$ are obtained by solving (16) at the vertices of the polytopic model (18)-(19).

The advantage of this approach is that the optimization problem (16) is solved offline. Thus, due to the limited available tuning matrices, an interpolation based on the estimated parameters is calculated to obtain the correct $\Lambda_{\mathrm{LMI}}^{*}$ with less computational effort. Consequently, the maximum number of optimization problems to be solved is then reduced to $N$. It is worth noting that solving the $\Lambda_{\mathrm{LMI}}^{*}$ online without interpolation has shown the same exact results of the proposed offline-solving of the optimization problem with online interpolation. However, the polytopic approach is preferable to employ, since it avoids time and computational memory consumption as already discussed.

2) Classical computation of $\Lambda$ (online): The minimization of the Frobenius norm of the generator matrix $H$ of a zonotope $\mathcal{X}$ using (4) leads to obtain an optimal tuning matrix $\Lambda^{*}$. It has also been proved that this online approach is independent of the weighting matrix [6], [10], [11]. Consequently, the optimal tuning matrix $\Lambda^{*}$ using the classical approach is computed online as:

$$
\Lambda^{*}=\frac{\hat{H} \hat{H}^{\top} C^{\top}}{C \hat{H} \hat{H}^{\top} C^{\top}+F_{v} F_{v}^{\top}} .
$$

In conclusion, the LMI-based approach is applicable to nonlinear systems, and only the interpolation of the feasible solutions in function of $\rho$ is computed online. Whereas, the classical approach is implemented online to linear systems.

\section{REMAINING USEFUL LIFE FORECASTING AND DECISION MAKING}

The desired outcome of the whole PHM application is a fast and reliable online RUL forecasting. Switching power electronic systems in specific, face harsh operating conditions. They may encounter stability issues such as unexpected behaviors, high temperatures or crashes. Moreover, the persistence of the robust systems is desired by maintaining the desired operational conditions. However, the degraded components have a slow and long-term impact on the efficiency of the system. As long as the faulty TH of the precursors are not crossed, it would be difficult to assess the health status of the system by direct measurements. Additionally, the scheduled maintenance might increase unnecessary burden and additional expenses. The RUL forecasting of slowdegraded power electronic systems is challenging due to many factors such as the complex modeling of the degradation and its integration. Therefore, the RUL forecasting is achieved online after the JESP process:

- Step 1: Retrieve the intervals of the estimated parameters only and assign the lower and the higher bounds of each parameter as: $\left[\underline{\hat{H}}_{k}\left(\rho_{j}\right), \hat{\bar{H}}_{k}\left(\rho_{j}\right)\right], \quad \forall k \in \mathbb{N}$. where $j$ denotes the number of the parameter $\rho$ denoting the degradation precursors (i.e $R_{\mathrm{ON}}, \mathrm{ESR}$ ).

Assumption 5.1: In the absence of permanent faults or other information at $k=1$, the system is assumed $100 \%$ healthy. 
- Step 2: Define a system of equations that describes unknown-behavior polynomial degradation as:

$$
\left\{\begin{array}{l}
f_{1}\left(p_{1}, p_{2}, \ldots, k, \mathrm{TH}\right), \\
f_{2}\left(p_{1}, p_{2}, \ldots, k, \rho_{0}, \hat{\rho}\right) .
\end{array}\right.
$$

where $\left(p_{1}, p_{2}, \ldots\right)$ are the parameters of the equations. Next, the system is solved for each bound of the estimated parameters as:

$$
\begin{aligned}
\Delta \underline{\rho}_{j_{k}} & =\underline{\hat{H}}_{k}\left(\rho_{j}\right)-\rho_{j_{0}}:\left(\underline{f}_{1}, \underline{f}_{2}\right), \\
\Delta \bar{\rho}_{j_{k}} & =\hat{\bar{H}}_{k}\left(\rho_{j}\right)-\rho_{j_{0}}:\left(\bar{f}_{1}, \bar{f}_{2}\right) .
\end{aligned}
$$

Thus, the coefficients of the polynomial systems $\left(p_{1}, p_{2}, \ldots\right)$ are obtained based on the estimated zonotopic bounds.

- Step 3: The previously-obtained parameters of the polynomial system are used to solve Eq. (22) for $\Delta \rho_{j_{k}}=$ $\mathrm{TH}_{\rho_{j}}$ and return $k$ which defines in this case the estimated EoL. Hence, the bounded RUL are obtained as:

$$
\left\{\begin{array}{l}
\underline{\mathrm{RUL}}_{k}\left(\rho_{j}\right)=\hat{\operatorname{EoL}}_{k}\left(\rho_{j}\right)-k, \\
\overline{\operatorname{RUL}}_{k}\left(\rho_{j}\right)=\overline{\operatorname{EoL}}_{k}\left(\rho_{j}\right)-k,
\end{array}\right.
$$

- Step 4: A safe decision-making has been adopted in this study, based on the following proposition:

Proposition 5.2 (FCCF): The RUL of the system is the RUL of the FCCF. Technically, the EoL of the system is decided based on the component that reaches the threshold first.

It is worth noting that the significant advantage of the proposed forecasting approach is independent of the predefined degradation behaviors which are only used in simulation as source of measurements in the simulation.

\section{CASE STUDY}

\section{A. LPV Modeling of a Degraded DC-DC Converter}

A DC-DC converter in boost operation mode has been employed to feature the effectiveness of the proposed PHM approach based on the JESP by set-membership using zonotopes. The average model of the switched power electronics system has been developed and detailed in previous work [7], [9]. Thus, the augmented representation of the dynamical model has been transformed into an LPV representation due to the aforementioned observability and nonlinearities concerns. The presence of the empirical degradation models of the critical components allows the simulation of different degradation scenarios in order to analyze the correlated degradation effects. Two vital power electronic components have been degraded and integrated in the converter model at the same time. Hence, the ON-resistor $\left(R_{\mathrm{ON}}\right)$ is the adopted degradation precursor of the MOSFET, and the Equivalent Series Resistance $\left(\mathrm{ESR}_{\mathrm{o}}\right)$ denotes the precursor of the output capacitor, where both are considered as varying parameters. The empirical degradation is implemented for the sole reason of simulating a degraded case based on real measurements of accelerated-aged components [13], [14].

The converter is rated at $30 \mathrm{~kW}$ with the ideal values of the input voltage $v_{\text {in }}=200 \mathrm{~V}$, and the output current $i_{\mathrm{o}}=100 \mathrm{~A}$. $R_{\text {in }}=0.01 \Omega$ is the input resistance, $C_{\text {in }}=80 \mathrm{mF}$ is the input capacitance and $\mathrm{ESR}_{\mathrm{in}}=100 \mathrm{~m} \Omega$ is the $\mathrm{ESR}_{\text {in }}$ of the input capacitor. Moreover, the inductance is denoted by $L=146 \mu$ $\mathrm{H}$ and $R_{L}=5 \mathrm{~m} \Omega$ is the internal resistance of the inductor. It should be noted that all the aforementioned parameters are assumed as constant values. Furthermore, the following empirical models of the $\mathrm{ESR}_{\mathrm{O}}$ and $R_{\mathrm{ON}}$ are described in the following equations, and they are only used to simulate the degradation mode:

$$
\begin{aligned}
& \operatorname{ESR}(t) \approx 0.08805 e^{3.649 .10^{-5} t}-0.008749 e^{-0.001097 t}, \\
& R_{\mathrm{ON}}(t) \approx R_{\mathrm{ON}_{0}}+0.0003332 e^{0.0003331 t}
\end{aligned}
$$

with the initial value of the output capacitor resistance $\mathrm{ESR}_{\mathrm{o}}=80 \mathrm{~m} \Omega$, the output capacitance $C_{\mathrm{o}}=5 \mathrm{mF}$ and the internal resistance of MOSFET is $R_{\mathrm{ON}}=0.2 \Omega$.

The following equations describe the states, inputs and outputs vectors respectively:

$$
\begin{aligned}
& x_{k}=\left[\begin{array}{lllll}
v_{C_{\text {in }}} & i_{L} & v_{C_{\mathrm{o}}} & \mathrm{ESR}_{\mathrm{o}} & R_{\mathrm{ON}}
\end{array}\right]^{\top}, \\
& u=\left[\begin{array}{ll}
v_{\text {in }} & i_{o}
\end{array}\right]^{\top}, \quad y=\left[\begin{array}{ll}
i_{i n} & v_{o}
\end{array}\right]^{\top} .
\end{aligned}
$$

Hence, the degradation equations in (25) are implemented in the following average state-space matrices:

$$
\begin{aligned}
& A(\rho)=\left[\begin{array}{ccccc}
\frac{-1}{C_{\mathrm{in}} \times R_{i C_{\mathrm{in}}}} & \frac{-\mathrm{ESR}_{\mathrm{in}}}{C_{\mathrm{in}} \times R_{i} C_{\mathrm{in}}} & 0 & 0 & 0 \\
\frac{\mathrm{ESR}_{\mathrm{in}}}{L \times R_{i C_{\mathrm{in}}}} & \frac{a_{22}}{L \times R_{i C_{\mathrm{in}}}} & \frac{d-1}{L} & a_{24} & a_{25} \\
0 & \frac{-(d-1)}{C_{o}} & 0 & 0 & 0 \\
0 & 0 & 0 & 1 & 0 \\
0 & 0 & 0 & 0 & 1
\end{array}\right] \text {, } \\
& B(\rho)=\left[\begin{array}{cc}
\frac{1}{C_{\mathrm{in}} \times R_{i} C_{\mathrm{in}}} & 0 \\
\frac{\mathrm{ESR}_{\mathrm{in}}}{L \times R_{i C_{\mathrm{in}}}} & \frac{-\mathrm{ESR}_{\mathrm{o}}(d-1)}{L} \\
0 & \frac{-1}{C_{o}} \\
0 & 0 \\
0 & 0
\end{array}\right], \\
& C(\rho)=\left[\begin{array}{ccccc}
\frac{-1}{R_{i C_{\text {in }}}} & \frac{\mathrm{ESR}_{\text {in }}}{R_{i C_{\text {in }}}} & 0 & 0 & 0 \\
0 & -\mathrm{ESR}_{\mathrm{o}}(d-1) & 1 & c_{24} & 0
\end{array}\right] \text {, } \\
& D(\rho)=\left[\begin{array}{cc}
\frac{1}{R_{i C_{\mathrm{in}}}} & 0 \\
0 & -\mathrm{ESR}_{\mathrm{o}}
\end{array}\right] \text {, }
\end{aligned}
$$

The distribution matrices of the noises and disturbances are:

$$
\begin{aligned}
E_{w} & =\operatorname{diag}(1,1,2,0.03,0.035), \\
E_{v} & =\operatorname{diag}(0.01,0.089) .
\end{aligned}
$$

Table I shows the variations on specific elements due to the transformation from a nonlinear to an LPV model. $M$ and $M^{\mathrm{LPV}}$ represent the nonlinear matrices, and the LPV model respectively. $d$ is the duty cycle of the switch. Henceforth, 
for the sake of simplicity, the varying states and parameters shown in the LPV model $i_{L}, \mathrm{ESR}_{\mathrm{O}}$ and $R_{\mathrm{ON}}$ will be denoted by $X_{2}, X_{4}$ and $X_{5}$ respectively.

TABLE I

Elements of the State-Space Matrices in the Nonlinear and LPV MODELS

\begin{tabular}{c||c|c} 
Elements & $\mathbf{M}$ & $\mathbf{M}^{\mathbf{L P V}}$ \\
\hline \hline $\mathbf{a}_{22}$ & $R_{i C_{\text {in }}\left(\left[\hat{\mathcal{X}}_{\mathbf{4}}\right](d-1)-R_{L}-\left[\hat{\mathcal{X}}_{\mathbf{5}}\right] \times d\right)-\mathrm{ESR}_{\text {in }} \times R_{\text {in }}}$ \\
$\mathbf{a}_{24}$ & 0 & {$\left[\hat{\mathcal{X}}_{\mathbf{2}}\right](d-1) / L$} \\
$\mathbf{a}_{25}$ & 0 & $-\left[\hat{\mathcal{X}}_{\mathbf{2}}\right] \times d / L$ \\
$\mathbf{c}_{\mathbf{2 4}}$ & 0 & $-\left[\hat{\mathcal{X}}_{\mathbf{2}}\right](d-1)$ \\
\hline
\end{tabular}

It should be noted that $\left[\hat{\mathcal{X}}_{2}\right],\left[\hat{\mathcal{X}}_{4}\right]$ and $\left[\hat{\mathcal{X}}_{5}\right]$ represent the estimated zonotopes and they are implemented in the model based on the zonotope inclusion property to satisfy the certain intersection where all the uncertainties are accounted and implemented in $\omega$ and $v$ [15].

\section{B. Results and Discussions}

The converter has been modelled and simulated in the accelerated degradation of the MOSFET and the DC-link capacitor which are represented by their degradation precursors (the $R_{\mathrm{ON}}$ and $\mathrm{ESR}_{o}$ ). Based on the aforementioned information, the interconnection of these components lead to the cascading degradation of the system itself. The system is then discretized using a sampling frequency of $50000 \mathrm{~Hz}$ to obtain satisfactory results. The duty cycle of the switch is $d=0.33$.

The ZSM has been implemented for the estimation of three states and two parameters with two measurements provided, in an LPV framework. The optimal classic and LMI-based approaches for observer tuning, have been also compared and illustrated. It should be noted that the estimation of the states is not illustrated due to size limitations. The parameters estimation and the RUL forecasting are illustrated. Table II shows the Relative Accuracy (RA) of all the states and parameters. To highlight the degradation effect, the first state $v_{C_{\text {in }}}$ shows a slight increase throughout the degradation process. Additionally, $i_{L}$ and $v_{C_{\mathrm{o}}}$ have been decreased by a total degradation of $5 \mathrm{~A}$ and $7 \mathrm{~V}$ respectively, due to the cascading damage.

Figure 2 clearly shows that the LMI-tuned set-membership approach provides a more accurate estimation. Both tuning matrices are optimal and guarantee the intersection. However, the classical approach closely encloses the higher bound to the empirical model.

The second augmented parameter and fault precursor is entitled as the fifth state is the $R_{\mathrm{ON}}$. The intersection is guaranteed and both approaches perform well with a small difference in terms of bounds.

The previously illustrated results are evaluated by calculating the average $\overline{\mathrm{RA}}$ of the estimated bounds using the two proposed approaches as: $\overline{\mathrm{RA}}=100 \times\left(\left|\hat{c}-c_{\text {(emp) }}\right|\right) / c_{\text {(emp), }}$, where $\hat{c}$ and $c_{\text {(emp) }}$ represent the estimated and the empirical

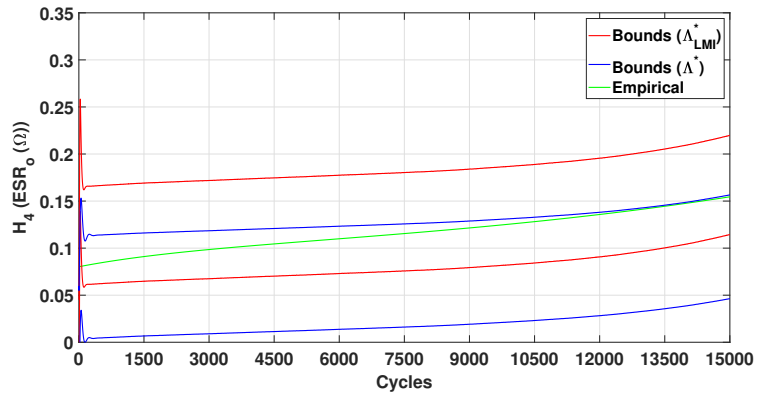

Fig. 2. Estimated parameter 4 showing the bounds tuned by two approaches

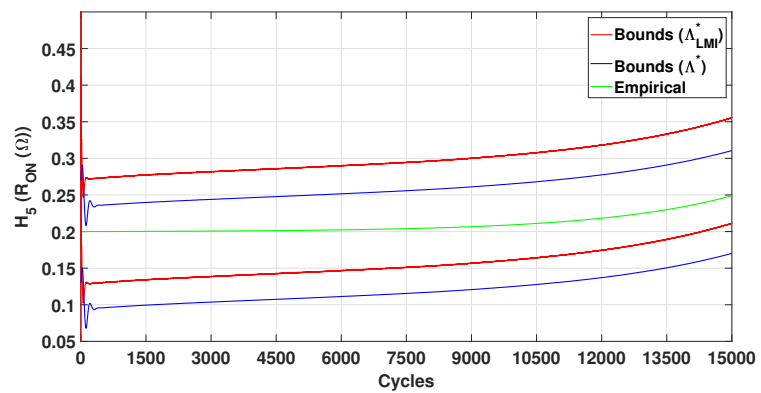

Fig. 3. Estimated parameter 5 showing the bounds tuned by two approaches

center of each zonotope respectively. Thus, the following table shows the average values of the RA of the three states and the two parameters.

TABLE II

The Average Relative Accuracy of the Estimated States and PARAMETERS

\begin{tabular}{c|c|c} 
States \& Parameters & $\operatorname{RA}\left(\Lambda_{\text {LMI }}^{*}\right) \%$ & $\operatorname{RA}\left(\Lambda^{*}\right) \%$ \\
\hline \hline $\mathcal{X}_{1}$ & 99.49522 & 99.49519 \\
$\mathcal{X}_{2}$ & 99.05189 & 99.05286 \\
$\mathcal{X}_{3}$ & 98.58109 & 98.47491 \\
$\mathcal{X}_{4}$ & 56.04974 & 54.2355 \\
$\mathcal{X}_{5}$ & 65.91433 & 66.65246
\end{tabular}

The average values of the RA of the estimated states and parameters using the two tuning matrices, are evidently matching with slight errors. Although the average RA of the two augmented parameters $\mathcal{X}_{4}$ and $\mathcal{X}_{5}$ are lower than the states, the intersection between the strip and the uncertain predicted states is always guaranteed. Consequently, the bounds always contain the empirical states and parameters, and the advantage of adopting the LMI-based approach over the online classical approach, is the ability of solving it offline with reduced computations.

Finally, the RUL algorithm has been applied online at the same time instant of each measurement and estimation. As previously stated, the RUL of the whole system is predicted based on the estimated parameters, ESR and $R_{\mathrm{ON}}$. The FCCF assumption is proposed in this paper, while the ongoing research for the predictive maintenance is being developed. Figure 4 shows the predicted RUL of each component 
using the two approaches of sets tuning compared to their respective empirical RUL. The LMI-based approach shows wider RUL intervals than the classical approach at each online forecasting. The results are provided in terms of intervals that converge towards the EoL. The RUL of the whole system is decided based on the lower RUL of a critical component which is the ESR of the DC-link capacitor as shown.

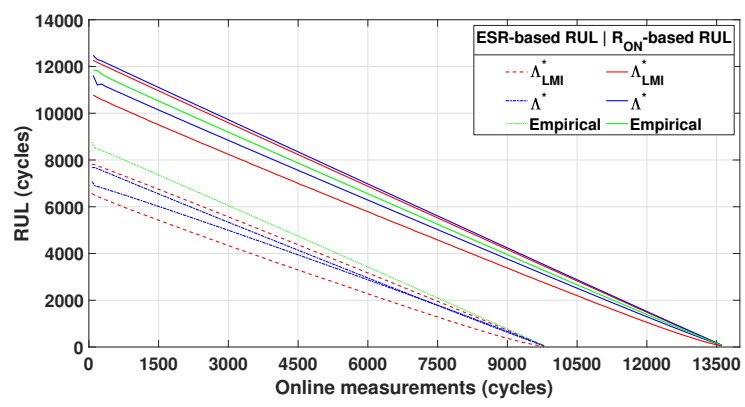

Fig. 4. RUL forecasting

Moreover, Table III shows a numerical example at an online measurement at 6000 cycles, featuring a comparison among the RUL forecasting based on each varying parameter, and the empirical RUL accordingly.

TABLE III

RUL FORECASTING INTERVALS AT AN ONLINE MEASUREMENT AT 6000 CYCLES

\begin{tabular}{cc|c|c|c}
\multicolumn{2}{c|}{ Critical Parameters } & $\underline{\text { RUL }}$ & $\hat{\text { RUL }}$ & Empirical RUL \\
\hline \hline \multirow{2}{*}{ ESR } & $\Lambda_{\text {LMI }}^{*}$ & 3666 & 4823 & 5233 \\
& $\Lambda^{*}$ & 4334 & 4584 & \\
\multirow{2}{*}{$R_{\mathrm{ON}}$} & $\Lambda_{\mathrm{LMI}}^{*}$ & 6194 & 7343 & 6 \\
& $\Lambda^{*}$ & 6725 & 7452 & 694
\end{tabular}

The most optimistic RUL interval at the online measurement at cycle 6000 is $[\underline{\mathrm{RUL}}, \hat{\mathrm{RUL}}]=[3666,4823]$ cycles. The LMI-based approach has been integrated in the forecasting algorithm for the robust results that has provided in the estimation process and later in the RUL.

\section{CONCLUSIONS}

This paper has proposed a robust ZSM-based PHM approach for RUL forecasting with an application to a DC-DC converter. The multi-aspect methodology contributes to the modeling of dynamical systems in an LPV framework, in phase I of the model-based PHM approach. Additionally, the ZSM observer has been employed in phase II for the JESP, that requires reduced computational efforts, and plays a crucial role in RUL forecasting. Thus, the ZSM observer has been optimally tuned for robust estimation in an offline LMI scheme which has shown encouraging results in comparison to the classical online approach. Hence, the proposed RUL forecasting algorithm estimates the EoL of each degraded component, and forecast their RUL bounds. Furthermore, our ongoing PHM proposition for multi-component-degraded systems is based on the FCCF proposition. The future work aims to employ the promising zonotopic-based techniques for RUL forecasting on a macro-level towards system-level prognostics.

\section{REFERENCES}

[1] S. Benmoussa and M. A. Djeziri, "Remaining useful life estimation without needing for prior knowledge of the degradation features," in IET Science, Measurement \& Technology, vol. 11, no. 8, pp. 10711078, 11 2017, doi: 10.1049/iet-smt.2017.0005.

[2] F. Schweppe, "Recursive state estimation: Unknown but bounded errors and system inputs," in IEEE Transactions on Automatic Control, vol. 13, no. 1, pp. 22-28, February 1968, doi: 10.1109/TAC.1968.1098790.

[3] M. Pourasghar, V. Puig and C. Ocampo-Martinez, "Robust Zonotopic Observer Design: Interval Observer versus Set-membership Approaches," 2019 4th Conference on Control and Fault Tolerant Systems (SysTol), Casablanca, Morocco, 2019, pp. 189-194, doi: 10.1109/SYSTOL.2019.8864760.

[4] V. T. H. Le, C. Stoica, T. Alamo, E. F. Camacho and D. Dumur, "Zonotope-based set-membership estimation for Multi-Output uncertain systems," 2013 IEEE International Symposium on Intelligent Control (ISIC), Hyderabad, 2013, pp. 212-217, doi: 10.1109/ISIC.2013.6658619.

[5] Ye Wang, Vicenç Puig, Gabriela Cembrano, Set-membership approach and Kalman observer based on zonotopes for discrete-time descriptor systems, Automatica, Volume 93, 2018, Pages 435-443, ISSN 00051098, https://doi.org/10.1016/j.automatica.2018.03.082.

[6] Christophe Combastel, Zonotopes and Kalman observers: Gain optimality under distinct uncertainty paradigms and robust convergence, Automatica, Volume 55, 2015, Pages 265-273, ISSN 0005-1098, https://doi.org/10.1016/j.automatica.2015.03.008.

[7] Ahmad Al-Mohamad, Ghaleb Hoblos, Vicenç Puig, A hybrid system-level prognostics approach with online RUL forecasting for electronics-rich systems with unknown degradation behaviors, Microelectronics Reliability, Volume 111, 2020, 113676, ISSN 0026-2714, https://doi.org/10.1016/j.microrel.2020.113676.

[8] Maarten Schoukens, Roland Tóth, Linear Parameter Varying Representation of a class of MIMO Nonlinear Systems, IFAC-PapersOnLine, Volume 51, Issue 26, 2018, Pages 94-99, ISSN 2405-8963, https://doi.org/10.1016/j.ifacol.2018.11.162.

[9] A. Al-Mohamad, V. Puig and G. Hoblos, "Zonotopic Extended Kalman Filter For RUL Forecasting With Unknown Degradation Behaviors," 2020 28th Mediterranean Conference on Control and Automation (MED), Saint-Raphäl, France, 2020, pp. 574-579, doi: 10.1109/MED48518.2020.9182829.

[10] T. Alamo, J.M. Bravo, E.F. Camacho, Guaranteed state estimation by zonotopes, Automatica, Volume 41, Issue 6, 2005, Pages 1035-1043, ISSN 0005-1098, https://doi.org/10.1016/j.automatica.2004.12.008.

[11] Ye Wang, Vicenç Puig, Gabriela Cembrano, Set-membership approach and Kalman observer based on zonotopes for discrete-time descriptor systems, Automatica, Volume 93, 2018, Pages 435-443, ISSN 00051098, https://doi.org/10.1016/j.automatica.2018.03.082.

[12] Yang, Shaoyong, D. Xiang, A. Bryant, P. Mawby, L. Ran and P. Tavner. "Condition Monitoring for Device Reliability in Power Electronic Converters: A Review." IEEE Transactions on Power Electronics 25 (2010): 2734-2752.

[13] J. Renwick, C. Kulkarni, and J Celaya "Capacitor Electrical Stress Data Set", NASA Ames Prognostics Data Repository (http://ti.arc.nasa.gov/project/prognostic-data-repository), NASA Ames Research Center, Moffett Field, CA

[14] J. R. Celaya, A. Saxena, S. Saha, and K. Goebel "MOSFET Thermal Overstress Aging Data Set", NASA Ames Prognostics Data Repository (http://ti.arc.nasa.gov/project/prognostic-data-repository), NASA Ames Research Center, Moffett Field, CA

[15] Montes de Oca, Saúl, Puig, Vicenç, Blesa, Joaquim, Robust fault detection based on adaptive threshold generation using interval LPV observers, International Journal of Adaptive Control and Signal Processing, Int. J. Adapt. Control Signal Process. Volume 26, Issue 3, 2012, Pages 258-283, ISSN 0890-6327, https://doi.org/10.1002/acs.1263 